\title{
$\mathrm{BMJ}$
}

\section{Economic evaluation of arthritis self management in primary care}

Anita Patel, senior lecturer, ${ }^{1}$ Marta Buszewicz, senior lecturer, ${ }^{2}$ Jennifer Beecham, principal research fellow,
professor of health and social care economics, ${ }^{3}$ Mark Griffin, lecturer,${ }^{2}$ Greta Rait, senior clinical lecturer, ${ }^{2,5}$
Irwin Nazareth, professor, ${ }^{2,5}$ Angela Atkinson, clinical trial officer, ${ }^{6}$ Julie Barlow, professor of health
psychology, research director, ${ }^{7}$ Andy Haines, director, professor of public health and primary care ${ }^{8}$

${ }^{1}$ Centre for the Economics of Mental Health, Institute of

Psychiatry, King's College London, London SE5 8AF

${ }^{2}$ Research Department of Primary Care and Population Health,

University College London School of Life and Medical Sciences

${ }^{3}$ Personal Social Services

Research Unit, London School of Economics and Political Science

${ }^{4}$ Personal Social Services

Research Unit, University of Kent, Canterbury, Kent

${ }^{5}$ MRC General Practice Research Framework, London

${ }^{6}$ North East Stroke Research Network, James Cook University Hospital, Middlesbrough

${ }^{7}$ Self-management Programme, Applied Research Centre in Health and Lifestyle Interventions,

Faculty of Health and Life

Sciences, Coventry University

${ }^{8}$ London School of Hygiene and

Tropical Medicine

Correspondence to: A Patel

anita.patel@iop.kcl.ac.uk

Cite this as: $B M J$ J 2009;339:b3532 doi:10.1136/bmj.b3532

\section{ABSTRACT}

Objective To assess the cost effectiveness of a self management programme plus education booklet for arthritis in primary care.

Design Cost effectiveness and cost utility analysis from health and social care and societal perspectives alongside a randomised controlled trial.

Setting 74 general practices in the United Kingdom. Participants 812 patients aged 50 or more with osteoarthritis of the hips or knees, or both, and pain or disability, or both.

Interventions Randomisation to either six sessions of an arthritis self management programme plus an education booklet (intervention group) or the education booklet alone (standard care control group).

Main outcome measures Total health and social care costs and total societal costs at 12 months; cost effectiveness (incremental cost effectiveness ratios and cost effectiveness acceptability curves) on basis of quality of life (SF-36, primary outcome measure), EuroQol visual analogue scale, and quality adjusted life years (QALYs).

Results At 12 months health and social care costs in the intervention group were $£ 101$ higher (95\% confidence interval £3 to £176) than those in the control group because the additional costs of the arthritis self management programme did not seem to be fully offset by savings elsewhere. There were no significant differences in societal costs (which were up to 13 times the size of health and social care costs) or any of the outcomes. From the health and social care perspective the intervention was dominated by the control on the basis of QALYs (which were non-significantly lower in the intervention group) and had incremental cost effectiveness ratios between $£ 279$ and $£ 13473$ for the other outcomes. From the societal perspective the intervention seemed superior to the control owing to nonsignificantly lower costs and non-significantly better outcomes on all measures except QALYs. Probabilities of the arthritis self management programme's cost effectiveness ranged between $12 \%$ and $97 \%$ (for thresholds ranging $£ 0$ to $£ 1000$ ) based on one point improvements in SF-36 outcomes, but the clinical significance of this is debatable. Probabilities of cost effectiveness on the basis of the visual analogue scale and QALYs were low.

Conclusions Cost effectiveness of an arthritis self management programme is not suggested on the basis of current National Institute for Health and Clinical Excellence cost perspectives and QALY thresholds. The probability of cost effectiveness is greater when broader costs and other quality of life outcomes are considered. These results suggest that the cost effectiveness of the Department of Health's expert patients programme cannot be assumed across all clinical conditions and that further rigorous evaluations for other conditions may be needed.

Trial registration Current Controlled Trials ISRCTN79115352.

\section{INTRODUCTION}

Osteoarthritis is one of the most common forms of arthritis, with at least 4.4 million people in the United Kingdom experiencing moderate or severe disease. ${ }^{1}$ Arthritis is one of the biggest causes of disability, costing the National Health Service and social services around $£ 5.5 \mathrm{bn}(\$ 9.1 \mathrm{bn} ; € 6.2 \mathrm{bn})$ per annum and leading to 206 million lost working days in 1999-2000. ${ }^{2}$ The condition is the second most common cause for days off work. An ageing population and longer working lives are likely to lead to increases in these societal impacts.

Evaluations of arthritis self management programmes in the United States suggest that these relatively inexpensive interventions can lead to a range of patient centred benefits and reductions in healthcare use. Although such studies provided the initial evidence base for England's expert patients programme, they provided limited evidence of cost effectiveness and had important methodological weaknesses. ${ }^{34}$

We assessed the cost effectiveness of a self management programme for arthritis compared with usual care within a large randomised trial based in UK primary care, using high quality and rigorous methodologies that would give meaningful results for both clinicians and health policy planners. 


\section{METHODS}

Full details of the study have been described elsewhere. ${ }^{5}$ Briefly, 812 patients aged 50 or more and with osteoarthritis of the hips, knees, or both, and pain or disability, or both, were recruited from 74 primary care practices across the UK. The patients were randomised to receive either six sessions of an arthritis self management programme plus an education booklet (intervention group) or the education booklet alone (control group). The self management programme was based on an existing course (Challenging Arthritis) provided by the voluntary organisation Arthritis Care. The booklet was designed for the study and incorporated information available to the public from Arthritis Care and the Arthritis Research Campaign. This reflects standard primary care, where giving written information is common practice.

The economic evaluation was undertaken from two perspectives: health and social care and societal. It focused on longer term cost effectiveness at the 12 month follow-up, although mean costs and outcomes at four months are also reported. Although the arthritis self management programme in this trial was provided outside the health and social care sector, we included it as a healthcare cost because the NHS is currently funding the implementation of such inter ventions. ${ }^{6}$

\section{Data collection}

An adapted version of the client service receipt inventory was used to collect economic data at individual level, ${ }^{78}$ administered as a self completion questionnaire alongside the other outcome measures at the baseline assessment before randomisation (in the general practice) and at the four month and 12 month follow-up (both by post). It focused on arthritis related resource use for the previous three months and, alongside personal characteristics, covered income and employment status; use of health and social care resources (drugs and hospital and community based health and social care); costs borne by participants, their family, and friends (out of pocket expenses, lost pay, and informal care); indirect impacts (time off work for participants and carers); and receipt of social security benefits.

\section{Unit costs}

To obtain three month costs for each participant at each assessment point we applied unit costs (see web extra on bmj.com) to data on resource use at individual level as recorded on the client service receipt inventory. We used estimates for national unit costs to reflect the fact that the study sample was drawn from practices around the UK and to facilitate generalisation of the findings.

We estimated the unit cost of the arthritis self management programme as an average cost per patient using a top-down approach in the absence of detailed information on the resources and costs absorbed by the programme. This estimate was based on rates paid by the trial to Arthritis Care for running the courses. Of 406 participants in the intervention group, seven could not be found places on a course in their area. For the 399 offered a place, $£ 65780$ was paid to Arthritis Care; an average cost of $£ 164.86$ per participant. This cost covered the course of six sessions, regardless of how many sessions were attended. We describe sensitivity analyses to explore the potential implications of this costing approach. The education booklet reflects standard practice in primary care and we assumed its costs to be included within our cost estimates for primary care.

Costs are reported in pounds sterling and are standardised to 2002-3 rates (the most recent available set of unit costs after the study ended) using, as appropriate, the NHS Executive's hospital and community health services inflation index or the personal social services inflation index. ${ }^{9}$ Approximations of 2007-8 rates can be made by multiplying costs by 1.2 (the inflation rate for hospital and community health services pay and prices $^{9}$. It was not necessary to discount costs or outcomes as the evaluation covered only one year.

\section{Outcomes}

The primary outcomes were the summary scores for the physical and mental health components of the short form health survey (SF-36). ${ }^{10}$ For the economic evaluation we also included the visual analogue scale and the five dimensional health state description of the EuroQol, the EQ-5D. ${ }^{11}$ All outcome measures were administered as self completion questionnaires during attendance for the baseline assessment and by post at the four and 12 month follow-ups. We applied utility weights from a UK general population survey ${ }^{12}$ to the EQ-5D health states to calculate utility values and quality adjusted life years (QALYs). QALYs were calculated using the total area under the curve approach, with linear interpolation between assessment points and baseline adjustment for comparisons. ${ }^{13}$ To calculate total QALYs for the one year follow-up period we summed the QALYs at four months (representing the period between the baseline and four month assessment) and at 12 months (representing the period between the four month and 12 month assessment).

\section{Analyses}

Data were double entered and analysed using SPSS for Windows Release 11.0.1 and Stata 8.2 for Windows.

Data on resource use from the client service receipt inventory formed the basis of the total cost calculation for each participant. If no report existed on the use of a particular resource on returned inventories, we assumed that it was not used. If participants reported on a resource but not the quantity used, we imputed this from within group means for participants with data for that item at the same assessment point. After this imputation for specific missing items we carried out inventory related analyses on available casesthat is, we excluded those with completely missing inventory data. Similarly, all five components of the EQ-5D are required to compute utilities and QALYs 
used in the economic evaluation. We imputed missing EQ-5D components (where up to four of the five possible dimensions were missing) for seven of $630(1.1 \%)$ cases at four months and seven of $599(1.2 \%)$ cases at 12 months using the last value carried forward method because integers are needed in the application of utility weights. We then carried out EQ-5D related analyses on available cases after this imputation.

Health and social care costs included the costs of health care, social care, and drugs. Societal costs were calculated as these health and social care costs plus the costs of informal care, social security benefit receipts, and out of pocket expenses of participants and their families and friends, lost pay, and time off work.

Costs and outcomes are reported as mean values with standard deviations. Mean differences between groups and 95\% confidence intervals were obtained by bootstrap regressions (5000 replications). The baseline value of the relevant item was added as a covariate for all comparisons of costs and outcomes at four and 12 months.

We assessed cost effectiveness using cost effectiveness acceptability curves. For each participant we calculated the net monetary benefit for a range of monetary values and presented the bootstrapped data graphically. ${ }^{14}$ The resulting curves show the probability of the intervention being cost effective compared with standard care for the selected range of monetary values. We calculated cost effectiveness acceptability curves for each of the four outcome measures from both cost perspectives. Incremental cost effectiveness ratios are also reported where both costs and outcomes were higher for either group.
For both approaches to assess cost effectiveness, we extrapolated costs at the 12 month follow-up (representing a retrospective three month period) to a one year period (that is, multiplied by four) to match the period reflected by the QALY data, under the assumption that these economic impacts were constant over the assessment period.

\section{Sensitivity analyses}

As the base case evaluation excluded cases without outcome data for the relevant analysis we assessed the impact of missing outcomes at four months and 12 months by imputing full samples. We did this by using the same hot decking and multiple imputation procedure (SOLAS For Missing Data Analysis version 3.2; Statistical Solutions) used elsewhere for the analysis of main study outcomes. ${ }^{5}$

We also undertook eight one way sensitivity analyses to assess the impact of various cost assumptions on the total health and social care and societal costs at 12 months. Firstly, we examined the impact of cost data being missing by imputing total costs using the hot decking procedure and multiple imputation procedure. We compared alternative cost effectiveness ratios on the basis of these imputed full sample costs and outcomes with the base case ratios.

Secondly, within the intervention group the base case evaluation includes 129 (32\%) participants who failed to attend any session of the arthritis self management programme and a further $58(14 \%)$ who attended only 1-3 sessions. Thus, nearly half did not receive a clinically significant "dose," defined as attending four

Table 1| Resource use at four months (for previous three month period) by patients assigned to an arthritis self management programme plus education booklet or the education booklet only (usual care, control)

\begin{tabular}{|c|c|c|c|c|c|}
\hline \multirow[b]{2}{*}{ Resource } & \multirow[b]{2}{*}{ Unit } & \multicolumn{2}{|c|}{ Intervention group } & \multicolumn{2}{|c|}{ Control group } \\
\hline & & Valid No & Mean (SD) & Valid No & Mean (SD) \\
\hline \multicolumn{6}{|l|}{ Hospital based services: } \\
\hline Admissions & Nights & 286 & $0.04(0.5)$ & 314 & $0.20(1.5)$ \\
\hline Outpatients & Appointments & 286 & $0.17(0.6)$ & 312 & $0.21(0.6)$ \\
\hline Accident and emergency & Visits & 286 & $0.02(0.2)$ & 314 & $0.03(0.2)$ \\
\hline Physiotherapy & Visits & 284 & $0.26(1.0)$ & 304 & $0.19(1.0)$ \\
\hline Occupational therapy & Visits & 288 & $0.07(0.4)$ & 319 & $0.03(0.2)$ \\
\hline \multicolumn{6}{|l|}{ Community based services: } \\
\hline General practitioner & Surgery visits & 278 & $0.90(1.4)$ & 292 & $0.94(1.3)$ \\
\hline General practitioner & Home visits & 289 & $0.02(0.1)$ & 323 & $0.04(0.4)$ \\
\hline General practitioner & Telephone calls & 288 & $0.08(0.5)$ & 319 & $0.10(0.5)$ \\
\hline Practice nurse & Surgery visits & 282 & $0.21(0.7)$ & 313 & $0.27(0.9)$ \\
\hline Practice nurse & Telephone calls & 293 & $0.03(0.2)$ & 321 & $0.01(0.1)$ \\
\hline Repeat prescription without general practitioner contact & Requests & 236 & $1.36(1.8)$ & 251 & $1.43(1.5)$ \\
\hline Meals on wheels & Meals & 292 & 0 & 324 & $0.28(5.0)$ \\
\hline Home help & Visits & 292 & $0.29(3.6)$ & 319 & $0.53(5.2)$ \\
\hline Social worker & Visits & 293 & $0.02(0.2)$ & 322 & $0.02(0.2)$ \\
\hline Social worker & Telephone calls & 292 & $0.01(0.1)$ & 323 & $0.07(0.6)$ \\
\hline Physiotherapist & Home visits & 293 & $0.03(0.4)$ & 319 & $0.04(0.7)$ \\
\hline Physiotherapist & Surgery or clinic visits & 281 & $0.29(1.2)$ & 297 & $0.26(1.0)$ \\
\hline Occupational therapist & Home visits & 290 & $0.01(0.1)$ & 321 & $0.02(0.2)$ \\
\hline \multicolumn{6}{|l|}{ Informal care: } \\
\hline Total care per average week & Hours & 294 & $5.80(11.4)$ & 327 & $7.1(14.5)$ \\
\hline
\end{tabular}


Table $2 \mid$ Resource use at 12 months (for previous three month period) by patients assigned to an arthritis self management programme plus education booklet or the education booklet only (usual care, control)

\begin{tabular}{|c|c|c|c|c|c|}
\hline \multirow[b]{2}{*}{ Resource } & \multirow[b]{2}{*}{ Unit } & \multicolumn{2}{|c|}{ Intervention group } & \multicolumn{2}{|c|}{ Control group } \\
\hline & & Valid No & Mean (SD) & Valid No & Mean (SD) \\
\hline \multicolumn{6}{|l|}{ Hospital based services: } \\
\hline Admissions & Nights & 275 & $0.11(1.1)$ & 301 & $0.52(4.3)$ \\
\hline Outpatients & Appointments & 273 & $0.28(0.7)$ & 300 & $0.26(0.9)$ \\
\hline Accident and emergency & Visits & 274 & $0.02(0.2)$ & 301 & $0.04(0.2)$ \\
\hline Physiotherapy & Visits & 263 & $0.29(1.1)$ & 283 & $0.18(0.7)$ \\
\hline Occupational therapy & Visits & 272 & $0.08(0.6)$ & 297 & $0.06(0.5)$ \\
\hline \multicolumn{6}{|l|}{ Community based services: } \\
\hline General practitioner & Surgery visits & 255 & $0.85(1.2)$ & 280 & $0.90(1.5)$ \\
\hline General practitioner & Home visits & 279 & $0.01(0.1)$ & 298 & $0.02(0.2)$ \\
\hline General practitioner & Telephone calls & 276 & $0.08(0.4)$ & 296 & $0.11(0.5)$ \\
\hline Practice nurse & Surgery visits & 268 & $0.19(0.9)$ & 290 & $0.20(0.9)$ \\
\hline Practice nurse & Telephone calls & 277 & $0.01(0.1)$ & 299 & $0.02(0.2)$ \\
\hline Repeat prescription without general practitioner contact & Requests & 223 & $1.46(1.7)$ & 245 & $1.62(1.8)$ \\
\hline Meals on wheels & Meals & 278 & $0.01(0.2)$ & 298 & 0 \\
\hline Home help & Visits & 277 & $0.85(11.0)$ & 295 & $0.57(5.8)$ \\
\hline Social worker & Visits & 278 & $0.03(0.3)$ & 297 & $0.05(0.3)$ \\
\hline Social worker & Telephone calls & 278 & $0.06(0.5)$ & 296 & $0.06(0.5)$ \\
\hline Physiotherapist & Home visits & 277 & $0.01(0.1)$ & 299 & $0.05(0.5)$ \\
\hline Physiotherapist & Surgery or clinic visits & 258 & $0.30(1.4)$ & 281 & $0.21(0.9)$ \\
\hline Occupational therapist & Home visits & 277 & $0.01(0.1)$ & 298 & $0.06(0.5)$ \\
\hline \multicolumn{6}{|l|}{ Informal care: } \\
\hline Total care per average week & Hours & 285 & $7.15(12.9)$ & 310 & $9.53(19.5)$ \\
\hline
\end{tabular}

or more sessions. ${ }^{15}$ Therefore the second sensitivity analysis was a per protocol analysis of costs based on those who attended four or more sessions.

The final six sensitivity analyses dealt with the average price charged to the trial by Arthritis Care as the unit cost of the arthritis self management programme. This price may not reflect the true economic cost per patient. For example, many of the activities of Arthritis Care are carried out by volunteers and the cost of their time is not included in the price. Moreover, the unit cost did not account for the number of sessions attended by each participant or the number present at each session. To deal with the various factors that would lead to an under-estimation or over-estimation, we varied the cost of the self management programme by $20 \%, 35 \%$, and $50 \%$ both upwards and downwards.

\section{RESULTS}

Response rates for the client service receipt inventory were $94 \%(n=765)$ at baseline, $77 \%(n=622)$ at four months, and $73 \%(\mathrm{n}=595)$ at 12 months and were balanced between the intervention and control groups. Corresponding response rates for the EQ-5D were $94 \%(\mathrm{n}=766), 78 \%(\mathrm{n}=630)$, and $74 \%(\mathrm{n}=599)$, which were also balanced between the groups.

\section{Resource use}

Evidence from the US on the purported economic advantages of self management interventions is commonly restricted to general practitioner visits, outpatient visits, and inpatient days. ${ }^{316}$ Utilisation rates for these services did not differ between the groups at baseline. At four months, general practitioner visits and outpatient visits remained similar (table 1), but fewer people in the intervention group (1.4\% v 2.9\%) had an inpatient stay, resulting in lower average inpatient days (table 1). A similar pattern was seen at 12 months (table 2). However, in both groups the trend was towards a reduction in general practitioner visits and an increase in outpatient visits compared with baseline. Hours spent on informal care were similar at all assessment points.

\section{Costs}

At baseline, health and social care costs or societal costs did not differ between the groups (table 3). At four months the intervention group had significantly higher costs from both perspectives. This is likely to be a direct consequence of the arthritis self management programme because when the costs of the programme were excluded a cost saving occurred in health and social care costs but no difference in societal costs.

Health and social care costs in the intervention group at 12 months were $£ 101$ higher $(95 \%$ confidence interval $£ 3$ to $£ 176$ ) than those in the control group. When the cost of the self management programme was reduced by between $20 \%$ and $50 \%$ in sensitivity analyses this difference became non-significant. Societal costs at 12 months were numerically, but not statistically, lower in the intervention group. This conclusion remained unchanged in all sensitivity analyses.

Although previous economic evaluations of self management interventions have focused on healthcare 
costs, and often for a limited range of services, healthcare costs formed a small proportion of societal costs (7\% at baseline). The largest single cost was social security benefits, at $44 \%$ of societal costs at baseline. Although benefits are often seen as a transfer cost, they represent high costs to the state. Costs borne by participants and their family and friends absorbed 37$46 \%$ of societal costs at each assessment point; mostly informal care. Costs for lost productivity were minimal, as most participants were not employed.

Table $3 \mid$ Costs ( $£, 2002-3$, three month periods), in base case and sensitivity analysis scenarios

\begin{tabular}{|c|c|c|c|c|c|}
\hline \multirow[b]{2}{*}{ Variables } & \multicolumn{2}{|c|}{ Intervention } & \multicolumn{2}{|c|}{ Control } & \multirow{2}{*}{$\begin{array}{l}\text { Intervention-control; mean } \\
\text { difference* }(95 \% \mathrm{Cl})\end{array}$} \\
\hline & Valid No & Mean (SD) & Valid No & Mean (SD) & \\
\hline Arthritis self management programme & 406 & $162(21)$ & 406 & 0 & $162(-160$ to -164$)$ \\
\hline \multicolumn{6}{|c|}{ Health and social care costs excluding self management programme: } \\
\hline Baseline & 384 & $82(136)$ & 381 & $84(138)$ & $-1(-21$ to 18$)$ \\
\hline 4 months & 295 & $84(144)$ & 327 & $115(282)$ & $-33(-69$ to -0.1$)$ \\
\hline 12 months & 285 & $112(240)$ & 310 & $172(717)$ & $-61(-159$ to 14$)$ \\
\hline \multicolumn{6}{|l|}{ Costs to patient, family, and friends: } \\
\hline Baseline & 384 & $510(1472)$ & 381 & $537(1075)$ & $-26(-199$ to 171$)$ \\
\hline 4 months & 294 & 479 (1073) & 327 & $453(845)$ & $22(-119$ to 163$)$ \\
\hline 12 months & 285 & $452(731)$ & 310 & $581(1094)$ & $-129(-270$ to 25$)$ \\
\hline \multicolumn{6}{|l|}{ Indirect costst: } \\
\hline Baseline & 384 & $13(113)$ & 381 & $43(361)$ & $-30(-70$ to 2$)$ \\
\hline 4 months & 295 & $15(148)$ & 327 & $20(228)$ & $2(-22$ to 27$)$ \\
\hline 12 months & 285 & $48(469)$ & 310 & $35(318)$ & $22(-45$ to 95$)$ \\
\hline \multicolumn{6}{|l|}{ Social security benefits: } \\
\hline Baseline & 384 & $490(614)$ & 381 & $510(633)$ & $-19(-108$ to 66$)$ \\
\hline 4 months & 295 & $620(690)$ & 327 & $545(628)$ & $74(-17$ to 160$)$ \\
\hline 12 months & 285 & $668(609)$ & 310 & $699(783)$ & $-28(-133$ to 76$)$ \\
\hline \multicolumn{6}{|c|}{ Total costs, health and social care perspective: } \\
\hline Baseline & 384 & $82(136)$ & 381 & $84(138)$ & $-1(-21$ to 18$)$ \\
\hline 4 months $\ddagger$ & 295 & $246(146)$ & 327 & $115(282)$ & 129 (93 to 162$)$ \\
\hline 12 months $\ddagger$ & 285 & $274(241)$ & 310 & $172(717)$ & 101 (3 to 176$)$ \\
\hline \multicolumn{6}{|l|}{ Total costs, societal perspective: } \\
\hline Baseline & 384 & $1096(1648)$ & 381 & $1173(1509)$ & $-77(-295$ to 153$)$ \\
\hline 4 monthsł & 294 & $1360(1459)$ & 327 & 1133 (1291) & $238(55$ to 434$)$ \\
\hline 12 months $\ddagger$ & 285 & $1442(1322)$ & 310 & 1487 (1918) & $-26(-277$ to 229$)$ \\
\hline \multicolumn{6}{|c|}{ Sensitivity analyses on total health and social care cost at 12 monthsł: } \\
\hline Imputed full sample§ & 406 & $259(363)$ & 406 & $183(683)$ & $77(-2$ to 155$)$ \\
\hline Per protocol analysis & 185 & $272(234)$ & 310 & $172(717)$ & 101 (-1 to 178$)$ \\
\hline $20 \%$ increase in ASMP cost & 285 & $307(242)$ & 310 & $172(717)$ & 133 (35 to 208) \\
\hline $35 \%$ increase in ASMP cost & 285 & $331(242)$ & 310 & $172(717)$ & 158 (59 to 233$)$ \\
\hline $50 \%$ increase in ASMP cost & 285 & $355(243)$ & 310 & $172(717)$ & 182 (84 to 257 ) \\
\hline $20 \%$ decrease in ASMP cost & 285 & $242(241)$ & 310 & $172(717)$ & $69(-30$ to 144$)$ \\
\hline $35 \%$ decrease in ASMP cost & 285 & $218(241)$ & 310 & $172(717)$ & $44(-54$ to 119$)$ \\
\hline $50 \%$ decrease in ASMP cost & 285 & $193(240)$ & 310 & $172(717)$ & $20(-78$ to 95$)$ \\
\hline \multicolumn{6}{|c|}{ Sensitivity analyses on total societal cost at 12 monthsł: } \\
\hline Imputed full sample§ & 406 & $1456(1398)$ & 406 & $1494(1851)$ & $-34(-270$ to 202$)$ \\
\hline Per protocol analysis & 185 & $1421(1319)$ & 310 & 1487 (1918) & -10 (-286 to 256$)$ \\
\hline $20 \%$ increase in ASMP cost & 285 & 1475 (1322) & 310 & 1487 (1918) & $6(-244$ to 261$)$ \\
\hline $35 \%$ increase in ASMP cost & 285 & $1499(1322)$ & 310 & 1487 (1918) & $31(-220$ to 286$)$ \\
\hline $50 \%$ increase in ASMP cost & 285 & $1523(1322)$ & 310 & 1487 (1918) & $55(-195$ to 310$)$ \\
\hline $20 \%$ decrease in ASMP cost & 285 & $1410(1322)$ & 310 & 1487 (1918) & $-58(-309$ to 197$)$ \\
\hline $35 \%$ decrease in ASMP cost & 285 & $1386(1322)$ & 310 & 1487 (1918) & $-83(-333$ to 173$)$ \\
\hline $50 \%$ decrease in ASMP cost & 285 & $1361(1322)$ & 310 & 1487 (1918) & $-107(-358$ to 149$)$ \\
\hline
\end{tabular}

ASMP=arthritis self management programme.

*Except for imputed full sample data, mean differences and confidence intervals were calculated using bootstrap regressions (5000 replications) with baseline scores as covariates for all values at four and 12 months.

†Time off work (participants and carers).

flncludes cost of ASMP.

§Missing data imputed using hot decking and multiple imputation procedures. Differences in means are adjusted for any baseline differences in costs between groups using analysis of covariance, with baseline cost as covariate. Confidence intervals were calculated using standard parametric techniques. 
_ SF-36 mental health - societal perspective

- - - SF-36 mental health - health and social care perspective

..--- SF-36 physical health - societal perspective

-- SF-36 physical health - health and social care perspective

- - VAS - societal perspective

- VAS - health and social care perspective

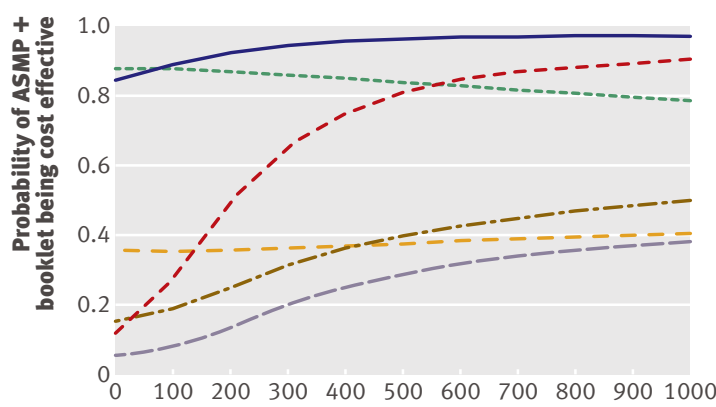

Willingness to pay for point gain on SF-36 and VAS (f)

Fig 1| Cost effectiveness acceptability curve of an arthritis self management programme (ASMP) on SF-36 and visual analogue scale (VAS) outcomes

\section{Outcomes}

At 12 months no significant differences were shown for the SF-36 summary scores, visual analogue scale, and QALYs (table 4). Confidence intervals suggested that the intervention group had marginally lower EQ-5D scores at four months; this difference did not remain in the imputed full sample analysis. Although there were some variations in mean differences for other outcomes computed using the available case and the imputed full sample approaches, the overall interpretation of results did not differ.

\section{Cost effectiveness}

The highest probabilities of cost effectiveness were for the two SF-36 outcomes from the societal perspective, for which probabilities were high even at low thresholds of willingness to pay for extra point gains (fig 1). From the health and social care perspective, however, probabilities of cost effectiveness on the basis of the mental health component exceeded 50\% (that is, the intervention was more likely to the cost effective option) only at willingness to pay levels of $£ 300$ or more per point gain and did not exceed 50\% for the physical health component for the threshold range examined. Probabilities of cost effectiveness on the basis of the visual analogue scale did not exceed $41 \%$ from either perspective for the threshold range examined. Probabilities of cost effectiveness based on QALYs were low, not exceeding $2 \%$ from the health and social care perspective even at thresholds of $£ 30000$ per QALY gain and peaking at $20 \%$ from the societal perspective for the threshold range examined (fig 2).

The meaningfulness of incremental cost effectiveness ratios is unclear given the lack of statistically significant differences for most combinations of costs and outcomes. Incremental cost effectiveness ratios from the health and social care perspective at 12 months were $£ 279$ for the SF-36 mental health component,

\begin{tabular}{|c|c|c|c|c|c|c|}
\hline \multirow[b]{2}{*}{ Scenarios } & \multicolumn{2}{|c|}{ Intervention } & \multicolumn{2}{|c|}{ Control } & \multicolumn{2}{|c|}{ Adjusted difference in means (intervention-control) } \\
\hline & Valid No & Mean (SD) & Valid No & Mean (SD) & Mean difference* $(95 \% \mathrm{Cl})$ & $\begin{array}{l}\text { Imputed full sample†: mean } \\
\text { difference }(95 \% \mathrm{Cl})\end{array}$ \\
\hline \multicolumn{7}{|c|}{ SF-36 physical health summary score: } \\
\hline Baseline & 316 & $25.59(11)$ & 317 & $25.35(12)$ & $0.24(-1.56$ to 2.03$)$ & \\
\hline 4 months & 234 & $25.98(12)$ & 268 & $25.66(13)$ & $0.12(-1.48$ to 1.49$)$ & $0.22(-1.50$ to 1.94$)$ \\
\hline 12 months & 231 & $25.62(12)$ & 252 & $25.18(12)$ & $0.34(-1.35$ to 2.00$)$ & $0.33(-1.31$ to 1.98$)$ \\
\hline \multicolumn{7}{|c|}{ SF-36 mental health summary score: } \\
\hline Baseline & 316 & $51.79(11)$ & 317 & $50.53(10)$ & $1.26(-0.38$ to 2.89$)$ & \\
\hline 4 months & 234 & $51.40(11)$ & 268 & $50.14(11)$ & $0.34(-1.25$ to 1.92$)$ & $0.11(-1.18$ to 1.40$)$ \\
\hline 12 months & 231 & $52.28(11)$ & 252 & $50.32(10)$ & $1.45(-0.17$ to 3.04$)$ & $1.35(-0.03$ to 2.74$)$ \\
\hline \multicolumn{7}{|c|}{ EuroQol visual analogue scale: } \\
\hline Baseline & 347 & $63.15(17)$ & 359 & $63.43(17)$ & $-0.28(-2.73$ to 2.27$)$ & \\
\hline 4 months & 269 & $64.41(17)$ & 303 & $63.16(18)$ & $1.32(-1.01$ to 3.66$)$ & $1.72(-0.32$ to 3.76$)$ \\
\hline 12 months & 242 & $63.62(18)$ & 273 & $62.36(17)$ & $0.03(-2.34$ to 2.40$)$ & $0.73(-1.38$ to 2.85$)$ \\
\hline \multicolumn{7}{|l|}{ EQ-5D utility score: } \\
\hline Baseline & 381 & $0.570(0.25)$ & 375 & $0.535(0.28)$ & $0.03(-0.00$ to 0.07$)$ & \\
\hline 4 months & 299 & $0.552(0.28)$ & 331 & $0.556(0.27)$ & $-0.04(-0.07$ to -0.00$)$ & $-0.03(-0.06$ to 0.01$)$ \\
\hline 12 months & 285 & $0.578(0.25)$ & 312 & $0.559(0.27)$ & $-0.005(-0.04$ to 0.03$)$ & $-0.006(-0.04$ to 0.03$)$ \\
\hline \multicolumn{7}{|l|}{ QALYs: } \\
\hline Baseline to 4 months & 290 & $0.191(0.08)$ & 316 & $0.183(0.08)$ & $-0.006(-0.01$ to -0.00$)$ & $-0.003(-0.01$ to 0.00$)$ \\
\hline 4-12 months & 256 & $0.383(0.15)$ & 291 & $0.373(0.16)$ & $-0.01(-0.03$ to 0.01$)$ & $-0.007(-0.03$ to 0.01$)$ \\
\hline Total for 1 year & 248 & $0.580(0.22)$ & 278 & $0.558(0.23)$ & $-0.01(-0.04$ to 0.01$)$ & $-0.01(-0.04$ to 0.01$)$ \\
\hline
\end{tabular}

QALYs=quality adjusted life years.

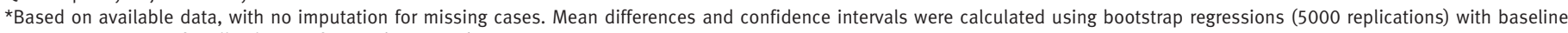
scores as covariates for all values at four and 12 months.

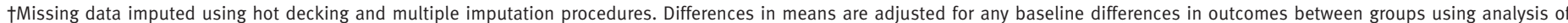
covariance, with baseline score as covariate. Confidence intervals were calculated using standard parametric techniques. 
$£ 1189$ for the SF-36 physical health component, and $£ 13473$ for the visual analogue scale. On the basis of these three outcomes, the intervention group dominated the control group from the societal perspective because of lower costs and better outcomes.

The QALY disadvantage to the intervention group, combined with higher health and social care costs, suggests that from the health and social care perspective the standard care approach was superior to the intervention. From the societal perspective, cost savings in the intervention group would need to be traded off against modest QALY losses, which would be a contentious policy decision. Thus neither the cost effectiveness acceptability curve nor incremental cost effectiveness ratio approach suggests that the intervention is cost effective compared with standard care on the basis of QALYs.

The broad conclusions of the approach on the basis of the incremental cost effectiveness ratio did not change with the use of imputed full sample data. Ratios remained similar except that the additional cost per point gain on the visual analogue scale from the health and social care perspective fell from $£ 13473$ to $£ 419$ as a result of a notable increase in mean difference in visual analogue scale score (0.03 $v 0.73$; see table 4$)$.

\section{DISCUSSION}

A self management programme for arthritis plus an education booklet in primary care led to a statistically significant increase in health and social care costs but no difference in societal costs or any of the measured outcomes at 12 months.

Although the lack of statistical significance for some differences in costs and outcomes could be deemed to weaken the cost effectiveness evidence, the inference approach has limitations. ${ }^{17}$ So it is nevertheless relevant to explore the implications for cost effectiveness. Some small advantages on the SF-36 outcomes translated into low incremental cost effectiveness ratios from the health and social care perspective and dominance from the societal perspective, as well as reasonably good probabilities of cost effectiveness for the intervention under the cost effectiveness acceptability curve approach. Findings on the basis of QALYs were less favourable, however: the intervention was dominated by the control from the health and social care perspective and involved a trade-off between slightly reduced QALYs and cost savings from the societal perspective. The cost effectiveness acceptability curve approach showed low probabilities of cost effectiveness even when high values were placed on QALY gains.

An important finding was the small proportion of support costs falling to the NHS and social care sectors. Three month costs for health and social care services were around $£ 80$ at baseline. Other societal costs were almost 13 times that, illustrating the breadth of the economic impacts of arthritis outside of these sectors. Furthermore, the probabilities of cost effectiveness were generally higher from a societal perspective, indicating that economic benefits of this intervention are likely to be borne more by participants, their families,

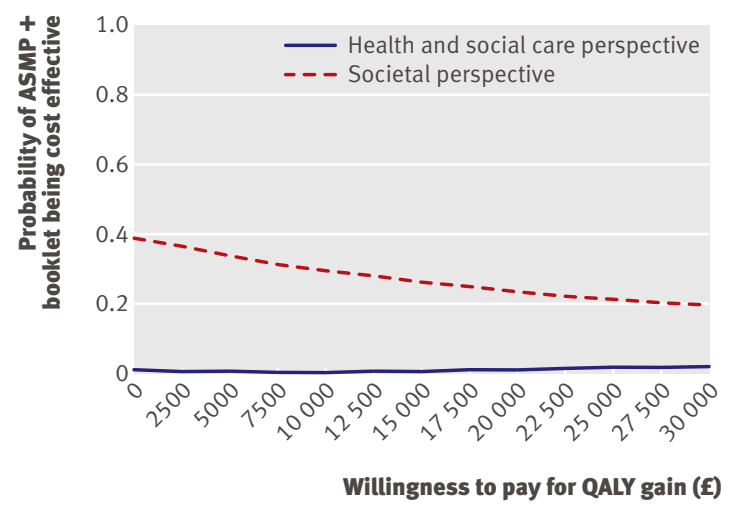

Fig 2 Cost effectiveness acceptability curve of an arthritis self management programme (ASMP) on QALY gains

and friends and other government sectors. To join up with wider policy considerations, such as the role of carers in the long term management of people with chronic conditions, ${ }^{18}$ broad perspectives are needed in economic evaluations of interventions such as self management programmes for arthritis to assess their impact on all stakeholders, despite the additional methodological challenges that this brings. ${ }^{19}$

\section{Strengths and limitations of the study}

This comprehensive economic evaluation, set within a randomised controlled trial, was based on individual patient data and therefore deals with many of the limitations associated with previous evidence. Furthermore, it fills an important evidence gap; its findings are likely to have a wider application because the sample was recruited from across the UK and among patients who routinely attend primary care rather than participants who had specifically volunteered to take part in the intervention.

The inclusion of social security benefits in the evaluation from a societal perspective may be contentious because such costs are generally considered to be transfer payments. However, social security benefits are an important consideration in certain patient groups and when the economic criterion of equity is to be considered alongside that of efficiency in resource allocation. ${ }^{2021} \mathrm{We}$ thought that examining an intervention for a condition mainly endured by older people without accounting for such costs would miss an important dimension to public sector financing and decision making.

Some data from the client service receipt inventory were incomplete. Missing responses in self completed questionnaires are to be expected, and given the size and geographical spread of the sample and the breadth of the analytical perspective taken it would not have been feasible to collect these data from alternative sources. To make efficient use of the data provided, however, we imputed missing components.

The main limitation was that we were unable to measure accurately the costs of the arthritis self management programme. A bottom-up cost estimation, based in the setting where the programme is most 
likely to be delivered, especially if funded by health services, is necessary to examine the true cost effectiveness of the course. Ideally, costs under different set-up scenarios and from different perspectives of funders would also be examined. We were informed by Arthritis Care that the charge to primary care trusts in England at the time of the study was $£ 2500$ per course, occasionally discounted to $£ 1850$, and that the cost did not include any profit element. Group sizes of 1012 were reported, suggesting costs between $£ 154$ and $£ 250$ per participant. Our unit cost falls into the lower end of this range and is likely to be an underestimate because it did not cover factors such as failed courses and development costs. Furthermore, it is likely that implementation in the NHS would incur additional resources to coordinate the courses. Indeed, the Department of Health estimated the cost of its expert patients programme to be $£ 250$ per patient. ${ }^{6}$ However, a lower unit cost of $£ 123$ per participant has been reported elsewhere. ${ }^{4}$ What all estimates have in common is that they are based on throughput, which in turn may affect costs and cost effectiveness, as suggested by the finding from our sensitivity analyses that total health and social care costs would be equivalent to, rather than higher than, costs under a standard care approach if the unit cost of the arthritis self management programme fell by $20-50 \%$.

Aside from inflationary increases in prices, it is possible that costs and outcomes have changed since the completion of the trial owing to a range of other factors -for example, policy changes related to health care, social care, social security, and carers. However, as we carried out a controlled evaluation with balanced groups at baseline, we have no reason to believe that any such changes would have had a differential impact on the two groups. The findings should therefore continue to be relevant, particularly as osteoarthritis is a prevalent long term condition and there remains a government policy to include and expand the expert patients programme through the NHS.

\section{Comparison with previous studies}

Although a US study based on a primary care population reported no differences in clinical outcomes or use of healthcare resources at four months when comparing an arthritis self management programme with an arthritis self management programme book, ${ }^{22}$ the cost effectiveness of arthritis self management programmes against usual care was unproved. For example, a study that found cost savings and improvements in pain was based on decision modelling rather than individual patient data. ${ }^{23}$ Poor methodologies leading to a lack of good quality evidence were highlighted in a review of 10 studies offering education on arthritis self management. ${ }^{24}$ The review found that only three studies reported fewer physician visits and lower healthcare costs, whereas the remainder did not measure service use or costs.

Studies in other populations, however, have suggested that self management programmes can be cost effective. A well designed economic evaluation of the
English NHS expert patients programme, a self care programme for people with a range of self defined long term conditions, found small advantages in costs (mean difference -£27) and QALYs (mean difference 0.02) compared with a waiting list control. ${ }^{625}$ It is unclear why our study did not reach similar conclusions, particularly as our intervention cost was lower (£165 $v £ 250$ ), but differences in study design may have been contributing factors. For example, the evaluation of the expert patients programme covered a broader range of clinical conditions, recruited volunteers, and had no specific inclusion or exclusion criteria, and so may have included participants with greater capacity for benefit.

A longitudinal study in people with heart disease, lung disease, stroke, or arthritis examined a self management programme based on the generic principles of arthritis self management programmes and reported improvements in health status and reductions in healthcare costs at one and two years. ${ }^{16}$ Several factors should be considered in interpreting this finding. Firstly, the cost implications of the intervention were not considered within the framework of a formal economic evaluation on the basis of costs at individual level but were instead based on extrapolations of average costs for particular services (for example, changes in visits to the emergency room or doctor). Secondly, some extrapolations were based on non-significant changes in costs over time. Finally, total costs per patient were not estimated, making it difficult to gauge the intervention's overall cost effectiveness. Two other non-randomised studies of self management programmes reported cost offsets, but again the findings were based on extrapolations of study group means for a selection of services used, and cost effectiveness was not assessed. ${ }^{2627}$ Similar concerns were expressed in a recent review of evidence for self management across different diseases. ${ }^{3}$

\section{Implications for policy}

The outcome analysis showed statistically significant improvements for anxiety and perceived self efficacy to manage symptoms. ${ }^{5}$ This economic evaluation did not show such advantages for quality of life outcomes or costs. Following the current policy making stance of focusing on QALYs and health and social care costs, the findings reported here suggest that the arthritis self management programme intervention has a low probability of being cost effective. The intervention is more likely to be cost effective if a societal perspective is taken - one that includes the financial effects on patients and their families and friends - or greater value is placed on SF-36 outcomes than on QALYs. However, it must be borne in mind that the advantages to the intervention group on the SF-36 outcomes were small and the cost effectiveness acceptability curves are based on willingness to pay for one point advantages on the SF-36. The clinical significance of such small changes on the SF-36 is debatable. 


\section{WHAT IS ALREADY KNOWN ON THIS TOPIC}

Evaluations of arthritis self management programmes in the United States suggested that these relatively inexpensive interventions can lead to a range of patient centred benefits and reductions in healthcare use

Although such studies provided the initial evidence base for England's expert patients programme, they had narrow perspectives, methodological flaws, and uncertain applicability to the UK

\section{WHAT THIS STUDY ADDS}

Cost effectiveness of arthritis self management programmes is not suggested on the basis of current cost perspectives and QALY thresholds from the National Institute for Health and Clinical Excellence

The probability of cost effectiveness is greater when broader costs and other quality of life outcomes are considered

At $7 \%$ of societal costs, costs for health and social care constitute a small proportion of total economic costs in this patient group

Conclusions and needs for future research

The conclusions from this study depend on perceptions of the relative importance of health and social care and societal costs and the appropriate outcomes for this patient group. The study does not suggest cost effectiveness based on the health and social care cost perspective or implicit cost per QALY threshold currently used by the National Institute for Health and Clinical Excellence. However arthritis has broad impacts and we found that there may be modest cost effectiveness advantages for the arthritis self management programme when other major impacts such as informal care and social security benefits are taken into account alongside small gains of doubtful clinical relevance on the SF-36. Further rigorous appraisal of the cost effectiveness of the Department of Health's expert patients programme is warranted for other meaningful health outcomes and other clinical conditions.

We thank Angela Coulter and Melissa Pickering for their help in carrying out this trial; Lisa Cotterill, Gill Dorer, Nicky Fasey, Jeannett Martin, Claire Newland, Gloria Randall, and Tom Sensky for being on the trial steering committee; the Medical Research Council general practice framework nurses and general practices who were involved; and the participants. Contributors: MB, GR, MG, AP, JBm, JBW, and AH conceived, designed, and ran the trial. IN and $A A$ were involved in the ongoing management of the trial. AA supervised the data collection. MG carried out the multiple imputation analyses. AP carried out the economic evaluation and takes responsibility for the integrity of the data and the accuracy of the data analysis. All authors were responsible for drafting the paper, revising it critically for important intellectual content, and approving the final version. $\mathrm{MB}$ is guarantor.

Funding: This trial was funded by the UK Medical Research Council (grant No G9900306). The trial was initially funded to run for three years, from June 2000 to 31 May 2003. A supplement and extension extended the trial completion date to 31 May 2004. The UK Medical Research Council funded the trial, peer reviewed the protocol, and was represented on the trial steering committee. The funders had no role in the study design or management, data collection, analysis and interpretation, writing of the report, or preparation of the manuscript.

Competing interests: None declared.

Ethical approval: This study was approved by the multicentre research ethics committee of the London office before the trial started in 2000 (reference MREC/99/2/87). Approval was also obtained from the local research ethics committees of areas where patients were recruited to the trial.

1 Arthritis Research Campaign. Arthritis: the big picture. Chesterfield: arc, 2002.
2 Arthritis Care. Arthritis care fact sheet. London: Arthritis Care, 2007. www.arthritiscare.org.uk/PublicationsandResources/ Listedbytype/Factsheets (accessed June 2008).

3 Richardson G, Gravelle H, Weatherly H, Ritchie G. Cost-effectiveness of interventions to support self-care: a systematic review. Int J Technol Assess Health Care 2005;21:423-32.

4 Griffiths C, Foster G, Ramsey J, Eldridge S, Taylor S. How effective are expert patient (lay led) education programmes for chronic disease? BMJ 2007;334:1254-7.

5 Buszewicz M, Rait G, Griffin M, Nazareth I, Patel A, Atkinson A, et al. Self management of arthritis in primary care: randomised controlled trial. BMJ 2006;333:879-82.

6 Kennedy A, Reeves D, Bower P, Lee V, Middleton E, Richardson G, et al. The effectiveness and cost effectiveness of a national lay-led self care support programme for patients with long-term conditions: a pragmatic randomised controlled trial. J Epidemiol Community Health 2007;61:254-61.

7 Beecham J, Knapp M. Costing psychiatric interventions. In: Thornicroft G, Brewin C, Wing J, eds. Measuring mental health needs. Oxford: Oxford University Press, 1992.

8 Beecham J, Knapp M. Costing psychiatric interventions. In: Thornicroft G, Brewin C, Wing J, eds. Measuring Mental health needs, 2nd ed. Oxford: Oxford University Press, 2002.

9 Netten A, Curtis L. Unit costs of health and social care. Canterbury: PSSRU, University of Kent, 2003.

10 Ware J, Sherbourn C. The MOS, 36 item short-form health survey (SF36). I: conceptual framework and item selection. Med Care 1992;30:473-83.

11 EuroQol Group. EuroQol: a facility for the measurement of healthrelated quality of life. Health Policy 1990;16:199-208.

12 Dolan P, Gudex C, Kind P, Williams A. A social tariff for EuroQol: results from a UK population survey. Discussion paper 138. York: University of York, 1995

13 Manca A, Hawkins N, Sculpher MJ. Estimating mean QALYs in trialbased cost-effectiveness analysis: the importance of controlling for baseline utility. Health Econ 2005;14:487-96.

14 Fenwick E, Claxton K, Sculpher M. Representing uncertainty: the role of cost-effectiveness acceptability curves. Health Econ 2001;10:779-87.

15 Lorig KR, Holman HR. Arthritis self-management studies: a twelve year review. Health Educ Q 1993;20:17-28.

16 Lorig KR, Ritter P, Stewart AL, Sobel DS, William Brown B, Bandura A, et al. Chronic disease self-management program. 2-year health status and health care utilization outcomes. Med Care 2001;39:1217-23.

17 Claxton K. The irrelevance of inference: a decision-making approach to the stochastic evaluation of health care technologies. J Health Econ 1999;18:341-64.

18 Department of Health. Caring about carers. A national strategy for carers. London: DoH, 1999.

19 Drummond $\mathrm{M}$, Weatherly H, Ferguson B. Economic evaluations of health interventions. A broader perspective should include costs and benefits for all stakeholders. BMJ 2008;337:770-1.

20 Raftery J. Have "lunatics taken over the asylums?" The rising cost of psychiatric services in England and Wales, 1860-1986. In: Knapp M, ed. The economic evaluation of mental health care. Aldershot: Arena, 1995.

21 Richardson J. Why economic costs may not be of interest in a national health scheme; or, costs fairness and reverse order analysis. Centre for Health Program Evaluation, working paper 126. Melbourne: Monash University, 2001.

22 Solomon DH, Warsi A, Brown-Stevenson T, Farrell M, Gauthier S, Mikels D, et al. Does self-management education benefit all populations with arthritis? A randomised controlled trial in a primary care physician network. J Rheumatol 2002;29:362-8.

23 Kruger JMS, Helmick CG, Callahan LF, Haddix AC. Cost-effectiveness of the arthritis self-help course. Arch Intern Med 1998;158:1245-9.

24 Bodenheimer T, Lorig K, Holman H, Grumbach K. Patient selfmanagement of chronic disease in primary care. JAMA 2002;288:2469-75.

25 Richardson G, Kennedy A, Reeves D, Bower P, Lee V, Middleton E, et al. Cost effectiveness of the Expert Patients Programme (EPP) for patients with chronic conditions. J Epidemiol Community Health 2008;62:361-7.

26 Lorig KR, Sobel DS, Ritter PL, Laurent D, Hobbs M. Effect of a selfmanagement program on patients with chronic disease. Effect Clin Pract 2001;4:256-62.

27 Lorig KR, Sobel DS, Stewart AL, Brown BW, Bandura A, Ritter P, et al. Evidence suggesting that a chronic disease self-management program can improve health status while reducing hospitalisation: a randomized trial. Med Care 1999;37:5-14.

Accepted: 11 March 2009 